\title{
Dual-emission color-controllable nanoparticle based molecular imprinting ratiometric fluorescence sensor for the visual detection of Brilliant Blue
}

\author{
Qian Yang ${ }^{\mathrm{a}, \mathrm{b}}$, Jinhua $\mathrm{Li}^{\mathrm{b}}$, Xiaoyan Wang ${ }^{\mathrm{b}, \mathrm{c}}$, Hailong Peng ${ }^{\mathrm{a}, \mathrm{d}}$, Hua Xiong ${ }^{\mathrm{a}, *}$, Lingxin Chen ${ }^{\mathrm{b}, *}$ \\ ${ }^{a}$ State Key Laboratory of Food Science and Technology, Nanchang University, Nanchang, 330047, China \\ ${ }^{\mathrm{b}}$ CAS Key Laboratory of Coastal Environmental Processes and Ecological Remediation, Research Center for Coastal Environmental Engineering and Technology, Yantai \\ Institute of Coastal Zone Research, Chinese Academy of Sciences, Yantai, 264003, China \\ ${ }^{\mathrm{c}}$ School of Pharmacy, Binzhou Medical University, Yantai, 264003, China \\ ${ }^{\mathrm{d}}$ School of Resources, Environmental, and Chemical Engineering, Nanchang University, Nanchang, 330031, China
}

\section{A R T I C L E I N F O}

\section{Keywords:}

Molecular imprinting

Ratiometric fluorescence

Dual-emission nanoparticles

Brilliant Blue

Visual detection

\begin{abstract}
A B S T R A C T
Single-component dual-emission nanoparticles were synthesized by chelating the organic ligand 8-hydroxyquinoline (HQ) to the surface of $\mathrm{CdTe} / \mathrm{ZnS}$ quantum dots, namely $\mathrm{CdTe} / \mathrm{ZnQ}$, and were used to construct a novel mesoporous structured molecular imprinting ratiometric fluorescence sensor by facile one-pot sol-gel polymerization for the visual detection of Brilliant Blue. The $\mathrm{CdTe} / \mathrm{ZnQ}_{2}$ had bimodal fluorescence belonging to CdTe and $\mathrm{ZnQ}_{2}$ segments, respectively; significantly, the emission wavelength of CdTe was optimized to be 630 $\mathrm{nm}$ for the largest overlap with the absorption spectrum of Brilliant Blue. Consequently, fluorescence resonance energy transfer (FRET) efficiency was greatly enhanced, resulting in ideal determination. A favorable linearity toward Brilliant Blue was obtained within $0-1.0 \mu \mathrm{mol} \cdot \mathrm{L}^{-1}$ along with profuse color evolution from orange to yellowish orange to yellowish green to green, and a high detectability of $8.8 \mathrm{nmol} \cdot \mathrm{L}^{-1}$ was offered. Excellent recognition selectivity for Brilliant Blue over possibly coexistent food colorants was demonstrated, with a high imprinting factor of 7.1. Furthermore, endogenous Brilliant Blue was detected ranging from 0.21 to $41.03 \mathrm{mg} / \mathrm{kg}$ in six typical food samples with relative standard deviations lower than $3.5 \%$, and the results agreed well with that afforded by conventional methods. Using Brilliant Blue as a model, this dual-emission color-controllable nanoparticle based imprinting ratiometric fluorescence sensor provided promising perspectives for the highly selective and sensitive, rapid, visual detection of colored substances in complicated matrices.
\end{abstract}

\section{Introduction}

Molecular imprinting, mimicking the behavior of antibodies, aims to copolymerize template molecules into cross-linked matrices and subsequently remove the templates to finally create the molecularly imprinted polymers (MIPs) with tailor-made binding sites [1]. These binding sites are complementary to the target templates in shape, size and functional groups, and can further be used to recognize targets selectively [1]. Compared with the natural counterparts, MIPs can withstand harsher environmental conditions and show better chemical/ physical stability [2]. Thereupon, especially in the chemo/biosensing field, molecular imprinting has received widespread preference, by combining with various transduction mechanisms to transduce the specific binding events into measurable signals [3]. In the sensing field, fluorescence based imprinting sensors have become a research hotspot, owing to the combination of super sensitivity of fluorescence detection and excellent selectivity of MIPs via various ingenious construction strategies, by virtue of the intrinsic fluorescence of templates or exotic fluorescence source such as inorganic semiconductor quantum dots (QDs) [4,5].

QDs have been frequently embedded into fluorescent sensors owing to their good chemical stability, wide excitation peak but narrow symmetrical emission peak, color tenability etc., especially the CdTe QDs [6]. Nowadays, hydrophilic CdTe QDs are usually synthesized for the convenient applications in aqueous solution, but inevitably, the dissociation of $\mathrm{Cd}^{2+}$ would not only weaken the luminescence intensity but also endanger the environment and human health [7]. Therefore, core-shell structured QDs are preferred by in-situ growing an inorganic shell on the CdTe core, such as CdTe/ZnS QDs. The outer ZnS shell can efficiently eliminate the surface dangling bonds and defects, so that the fluorescence characteristics of emission wavelength and fluorescence quantum yield would change [4]. Additionally, compared with other core-shell QDs such as CdTe/CdS, the CdTe/ZnS QDs are of less toxicity and better biocompatibility by suppressing the $\mathrm{Cd}^{2+}$ release,

\footnotetext{
* Corresponding authors.

E-mail addresses: huaxiong100@126.com (H. Xiong), lxchen@yic.ac.cn (L. Chen).
} 
demonstrated by the $\mathrm{Cd}^{2+}$ dissociation test and hemolysis assay [8]

Generally speaking, the fluorescence detection is compromised to several analyte-independent factors such as background interference, instrumental fluctuation, operational parameters and so forth [9]. Fortunately, depending on the self-referencing effect, ratiometric fluorescence sensors containing target-sensitive and reference fluorescence substances can avoid these disadvantages [9]. Besides, different from the general analyte-sensitive fluorescence brightness variation of single-emission sensors, the ratiometric sensors can provide profuse color evolution depending on the change in fluorescence intensities ratio of the two emission peaks $[10,11]$. Therefore, ratiometric fluorescence sensors can easily realize the visual detection of target analytes by the naked eye, with the aid of a portable ultraviolet lamp but dispensing with large-scale fluorescent instruments. Typically, there are three construction strategies for the molecular imprinting ratiometric fluorescence sensors as below: the two-step-based core-shell structure of embedding the reference dye into cores and then anchoring imprinting shell on their surface in the presence of target-sensitive dye $[10,12]$; the time-saving one-step copolymerization of two different dyes in the imprinting procedure, whose optimization such as coupling or chemical modification is arduous [13]; synthesizing bicolor-emission nanoparticles and then doping them into MIPs, without the above problems [14]. Based on the CdTe/ZnS QDs mentioned above, bicoloremission nanoparticles can be proposed by chelating the surface $\mathrm{Zn}^{2+}$ ions with the functional ligand of 8-hydroxyquinoline (HQ), and then binding the resultant stable fluorescent HQ-metal complex, named $\mathrm{ZnQ}_{2}$ for simplicity (i.e., two HQ molecules reacting with one $\mathrm{Zn}^{2+}$ ion), to the surface via the dangling sulfide bond. Consequently, dual emission was endowed where one from the newly formed $\mathrm{ZnQ}_{2}$ complex and the other from the inherent CdTe QDs [15]. Hence, it is expected to construct molecular imprinting ratiometric fluorescence sensors depending on the single-component but dual-emission CdTe/ $\mathrm{ZnQ}_{2}$ nanoparticles for highly selective and sensitive detection of specific analytes with great monitoring significance, for instance, synthetic food colorants.

Synthetic food colorants have been used for imparting color to products for a long time in the food industry, to increase the sensorial attractiveness of foodstuffs toward consumers [16]. In comparison with the natural colorants, the synthetic compounds present less color loss against external conditions and lower cost of manufacture [16,17]. However, the synthetic colorants have numerous side effects on consumers' health especially when they are consumed in excess, such as toxicity, allergy, asthma, possible carcinogenesis and children's attention deficit hyperactivity disorder [18,19]. Hence, the regulatory legislations of stricter standards are adopted to control their usage. Taking Brilliant Blue for an example, its acceptable daily intake (ADI) was established as $12.5 \mathrm{mg} / \mathrm{kg}$ bw/day by the European Union (EU) Scientific Committee for Food (SCF) in 1975, and then European Food Safety Authority (EFSA) revised the ADI of $10 \mathrm{mg} / \mathrm{kg}$ bw/day allocated by the SCF in 1984 to be $6 \mathrm{mg} / \mathrm{kg}$ bw/day in 2010. And currently in the EU, Brilliant Blue is allowed in various foodstuffs with a maximal level of $20-500 \mathrm{mg} / \mathrm{kg}$, and in beverages less than $200 \mathrm{mg} / \mathrm{L}$ [20]. Meanwhile, more and more analytical methods have been utilized to measure the colorants' concentrations, involving photoacoustic spectroscopy [21], capillary electrophoresis [22], electrochemical determination [23], high-performance liquid chromatography (HPLC) [24], surface-enhanced Raman scattering [25], and so on. Nevertheless, to the best of our knowledge, molecular imprinting ratiometric fluorescence sensors have not been reported yet for synthetic food colorants detection.

Therefore, herein, we proposed a facile strategy to construct a mesoporous structured molecular imprinting ratiometric fluorescence sensor, based on the single-component dual-emission $\mathrm{CdTe} / \mathrm{ZnQ}_{2}$ nanoparticles and by simple one-pot sol-gel polymerization, for the visual detection of Brilliant Blue via fluorescence resonance energy transfer (FRET) mechanism. The as-constructed sensor was well characterized and explored for possible detection principle, as well as several variables were optimized and corresponding analytical performances were investigated systematically. The sensor was also applied for the determination of Brilliant Blue in various food samples with satisfactory results, indicating great application potential as a simple, highly selective and sensitive detection method for colorants in complicated matrices.

\section{Experimental section}

\subsection{Reagents and materials}

Brilliant Blue (called by China) namely Brilliant Blue FCF (E 133) (called by EU) was purchased from Solarbio Science \& Technology Co. Ltd. (Beijing, China), which was synthesized by condensation and oxidation reactions between benzaldehyde o-sulfonic acid and $\mathrm{N}$-ethyl- $\mathrm{N}$ (3-sulfobenzyl)-phenylamine, and its chemical structure was shown in Fig. S1. Indigotine was purchased from Solarbio Science \& Technology Co. Ltd. (Beijing, China). Gardenia Blue, Spirulina Blue and Sodium Copper Chlorophyllin, and several food samples (carbonated beverage, brandy cocktail, popping candy, chocolate candy, mung bean cake, and dried blueberry) were purchased from a local supermarket (Yantai, China). More details about Brilliant Blue, Gardenia Blue and Spirulina Blue were given in Supporting Information. Tellurium, sodium borohydride $\left(\mathrm{NaBH}_{4}\right)$ and thioglycolic acid (TGA) were offered by SigmaAldrich (Shanghai, China). Reduced glutathione (GSH), 8-hydroxyquinoline (HQ), 3-aminopropyl triethoxy silane (APTES), cetyltrimethylammonium bromide (CTAB), tetraethyl orthosilicate (TEOS) and Sunset Yellow were supplied by Aladdin (Shanghai, China). Chrome chloride $\left(\mathrm{CdCl}_{2} \cdot 5 \mathrm{H}_{2} \mathrm{O}\right)$, zinc chloride $\left(\mathrm{ZnCl}_{2}\right)$, sodium hydroxide $(\mathrm{NaOH})$, ammonium hydroxide $\left(\mathrm{NH}_{3} \cdot \mathrm{H}_{2} \mathrm{O}\right)$, ethanol and acetonitrile were provided by Sinopharm Chemical Reagent Co. Ltd. (Shanghai, China). All reagents were at least of analytical grade and used as received unless otherwise specified. Ultrapure water of $18.2 \mathrm{M} \Omega$ specific resistance (Millipore, USA) was used in all experiments.

\subsection{Instrumentation}

Fluorescence emission and UV-vis absorption spectra measurements were performed on a Fluoromax-4 Spectrofluorometer (Horiba Scientific) and a Thermo Scientific NanoDrop 2000/2000C Spectrophotometer (Thermo Fisher Scientific), respectively. HPLC-UV chromatograms were recorded by an Agilent 1260 Infinity HPLC system (Agilent Technologies) equipped with a diode array detector (DAD), and the test condition for Brilliant Blue was described in detail in Supporting Information. Morphological evaluation was examined on a scanning electron microscope (SEM, Hitachi S-4800), a transmission electron microscope (TEM, JEM-1230) and a high resolution transmission electron microscope (HRTEM, JEM-2100). Elemental mapping was observed by energy disperse spectroscopy (EDS, JSM-6701 F). FTIR analyses were carried out by a FT-IR spectrometer (Thermo Nicolet Corporation).

\subsection{Synthesis of dual-emission color-controllable nanoparticles of CdTe/ $Z n Q_{2}$}

Firstly, red-emitting TGA-modified CdTe QDs were prepared using an established procedure from our laboratory [26], with details mentioned in Supporting Information. Then, GSH-capped CdTe/ZnS QDs were synthesized by the situ growth of ZnS shell on the surface of CdTe core in aqueous solution [8]. Briefly, $8 \mathrm{~mL}$ of as-prepared CdTe sample was added to $17 \mathrm{~mL}$ aqueous solution containing $0.025 \mathrm{mmol} \mathrm{ZnCl}_{2}$ and $0.1 \mathrm{mmol} \mathrm{GSH}$. After being adjusted to $\mathrm{pH} 8$, the mixture was heated to $100{ }^{\circ} \mathrm{C}$ under open-air conditions and refluxed for certain time. Finally, bicolor-emission $\mathrm{CdTe} / \mathrm{ZnQ}_{2}$ nanoparticles were prepared by adding $50 \mu \mathrm{L} \mathrm{HQ}$ (in ethanol at different concentration) into $14 \mathrm{~mL}$ as-prepared $\mathrm{CdTe} / \mathrm{ZnS}$ under stirring for $15 \mathrm{~min}$ [27]. The color, i.e. the emission 


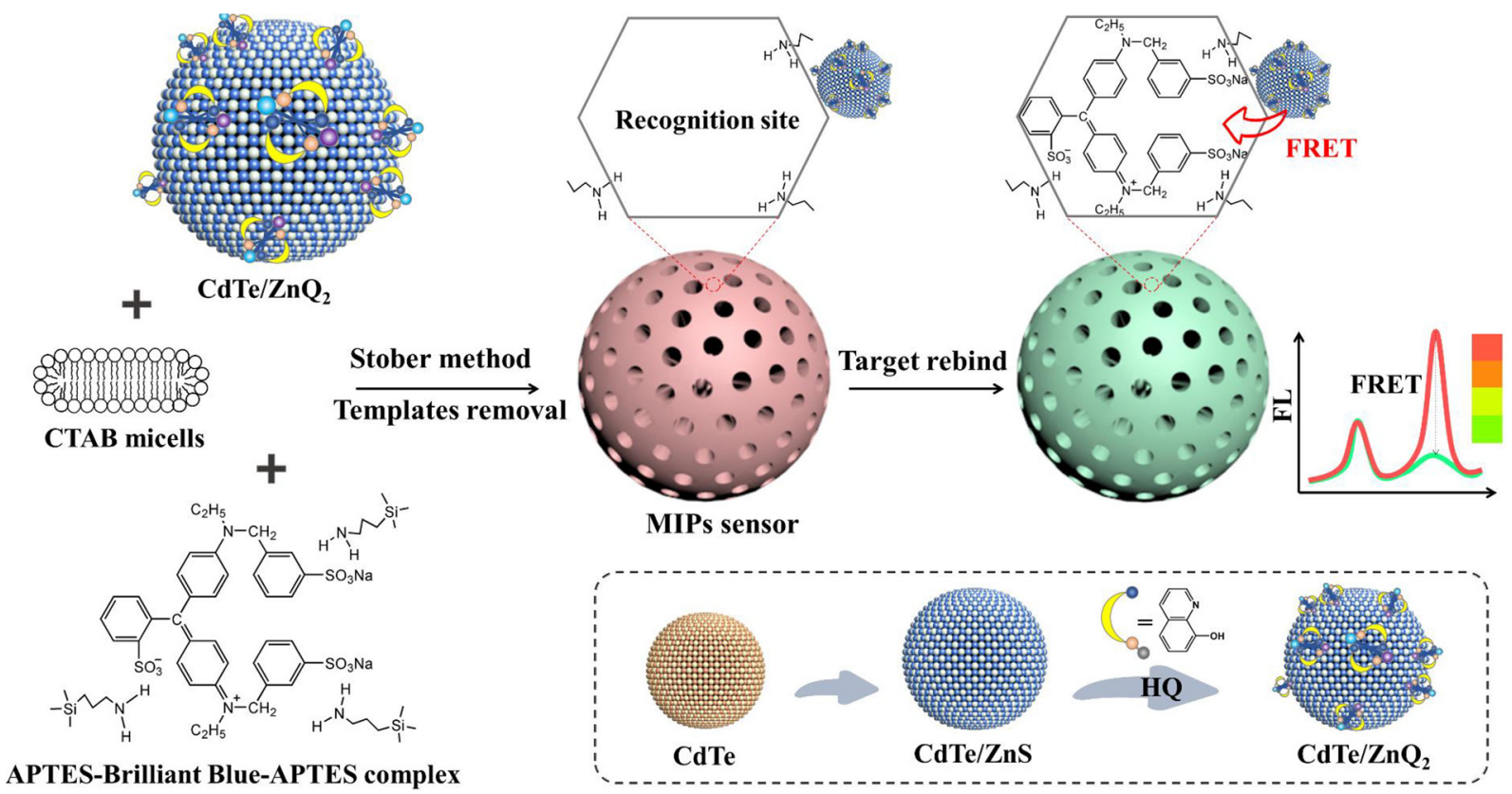

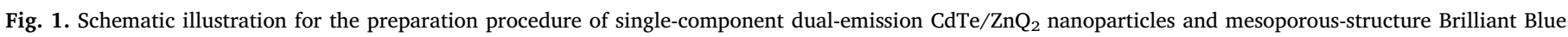

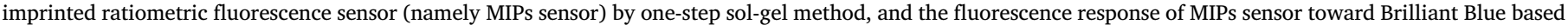
on the FRET mechanism. (For interpretation of the references to colour in this figure legend, the reader is referred to the web version of this article.)

wavelength, of the synthesized $\mathrm{CdTe} / \mathrm{ZnQ}_{2}$ nanoparticles was mainly controlled by the original emission of CdTe, the growth time of $\mathrm{ZnS}$ shell and the concentration of HQ.

\subsection{Construction of mesoporous structured Brilliant Blue imprinted ratiometric fluorescence sensor}

According to the reported one-pot sol-gel method [12], $3.2 \mathrm{mg}$ of Brilliant Blue and $37 \mu \mathrm{L}$ of APTES were added to the above $\mathrm{CdTe} / \mathrm{ZnQ}_{2}$ system and stirred for $1 \mathrm{~h}$. Then, $800 \mu \mathrm{L}$ of CTAB solution $\left(0.2 \mathrm{~mol} \cdot \mathrm{L}^{-1}\right)$, $100 \mu \mathrm{L}$ of $\mathrm{NaOH}\left(0.2 \mathrm{~mol} \cdot \mathrm{L}^{-1}\right), 178 \mu \mathrm{L}$ of TEOS, $178 \mu \mathrm{L}$ of $\mathrm{NH}_{3} \cdot \mathrm{H}_{2} \mathrm{O}$ and $4.657 \mathrm{~mL}$ water were injected successively and kept reaction overnight in darkness. After centrifugation, СTAB (template for mesoporous structure) and Brilliant Blue (template for recognition sites) were eluted from the imprinting matrix by ethanol/acetonitrile $(8: 2, \mathrm{v} / \mathrm{v})$ mixture under ultrasound assistance, using UV-vis and FT-IR spectrums to confirm their complete removal. Consequently, the mesoporous structured Brilliant Blue imprinted ratiometric fluorescence sensor was attained, namely mMIP@CdTe/ZnQ 2 , or MIPs sensor for simplicity. As control, mesoporous-structure imprinted polymers with single fluorescence emission (mMIP@CdTe/ZnS) were prepared in the same procedure but replacing $\mathrm{CdTe} / \mathrm{ZnQ}_{2}$ with the single-emission CdTe/ZnS QDs. For comparison, mesoporous-structure non-imprinted ratiometric fluorescence sensor (called NIPs for simplicity) was also synthesized according to the mMIP@CdTe/ZnQ 2 preparation procedure but without adding the template molecule of Brilliant Blue.

\subsection{Fluorescence measurements}

Fluorescence measurements were performed under the optimal conditions that $583 \mu \mathrm{g}$ MIPs or NIPs were firstly dispersed into $1 \mathrm{~mL}$ PBS solution ( $0.01 \mathrm{~mol} \cdot \mathrm{L}^{-1}, \mathrm{pH}$ 9) containing different concentrations of Brilliant Blue from 0 to $5 \mu \mathrm{mol} \cdot \mathrm{L}^{-1}$; after incubation for $6 \mathrm{~min}$ in the capped and dark tubes, the fluorescence spectra were recorded upon excitation at $365 \mathrm{~nm}$ with slit widths of $5 / 5 \mathrm{~nm}$. Specifically, the PBS buffer solution was stored in a sealed reagent bottle and placed in a refrigerator $\left(4^{\circ} \mathrm{C}\right)$, and re-prepared every two weeks. Hence, the sensing system would be exposed to $\mathrm{CO}_{2}$ in normal atmosphere for rather short time, which can ensure that dissolution of $\mathrm{CO}_{2}$ was negligible and the fluorescence studies at $\mathrm{pH} 9$ were highly effective, stable and reliable. The selectivity of the sensor was verified by its quenching efficiency toward other food colorants of Gardenia Blue, Indigotine, Spirulina Blue, Sodium Copper Chlorophyllin and Sunset Yellow at the same concentration of $1 \mu \mathrm{mol} \cdot \mathrm{L}^{-1}$ as Brilliant Blue.

\subsection{Analysis of food samples}

Six typical kinds of food samples were used to evaluate the practical application feasibility of the MIPs sensor. Referring to the PRC standard GB 5009.35 - 2016, the liquid food samples of fruity carbonated beverages and brandy cocktail were heated to remove the carbon dioxide and alcohol; the solid samples of hard candy, chocolate candy, mung bean cake and dried blueberry were pulverized and dissolved. And subsequently, these pretreated aqueous samples were adsorbed by polyamide and then desorbed by ethanol/ $\mathrm{NH}_{3} \cdot \mathrm{H}_{2} \mathrm{O}$ /water (700:4:100, $\mathrm{v} / \mathrm{v} / \mathrm{v})$ solution to extract the possible Brilliant Blue. After filtered through $0.22 \mu \mathrm{m}$ pore size membrane, concentrations of Brilliant Blue in these samples were detected by conventional HPLC-UV and UV-vis spectrophotometry. Taking into consideration the maximum permitted level of Brilliant Blue in various samples, proper volumes of these samples were added to the sensing system with final maximal level within the linear range of our constructed MIPs sensor. The practical application feasibility of the MIPs sensor was evaluated by comparing the obtained Brilliant Blue concentrations with those offered by HPLC$\mathrm{UV}$ and UV-vis.

\section{Results and discussion}

3.1. Construction and characterization of the dual-emission nanoparticles and MIPs sensor

The whole construction procedures of bicolor-emitting $\mathrm{CdTe} / \mathrm{ZnQ}_{2}$ nanoparticles and MIPs sensor are illustrated in Fig. 1. As displayed in the dashed rectangle in Fig. 1, hydrophilic CdTe/ZnS QDs were firstly synthesized through in situ growth of ZnS shell on the surface of TGAstabilized CdTe core in alkaline aqueous solution, using GSH as both 

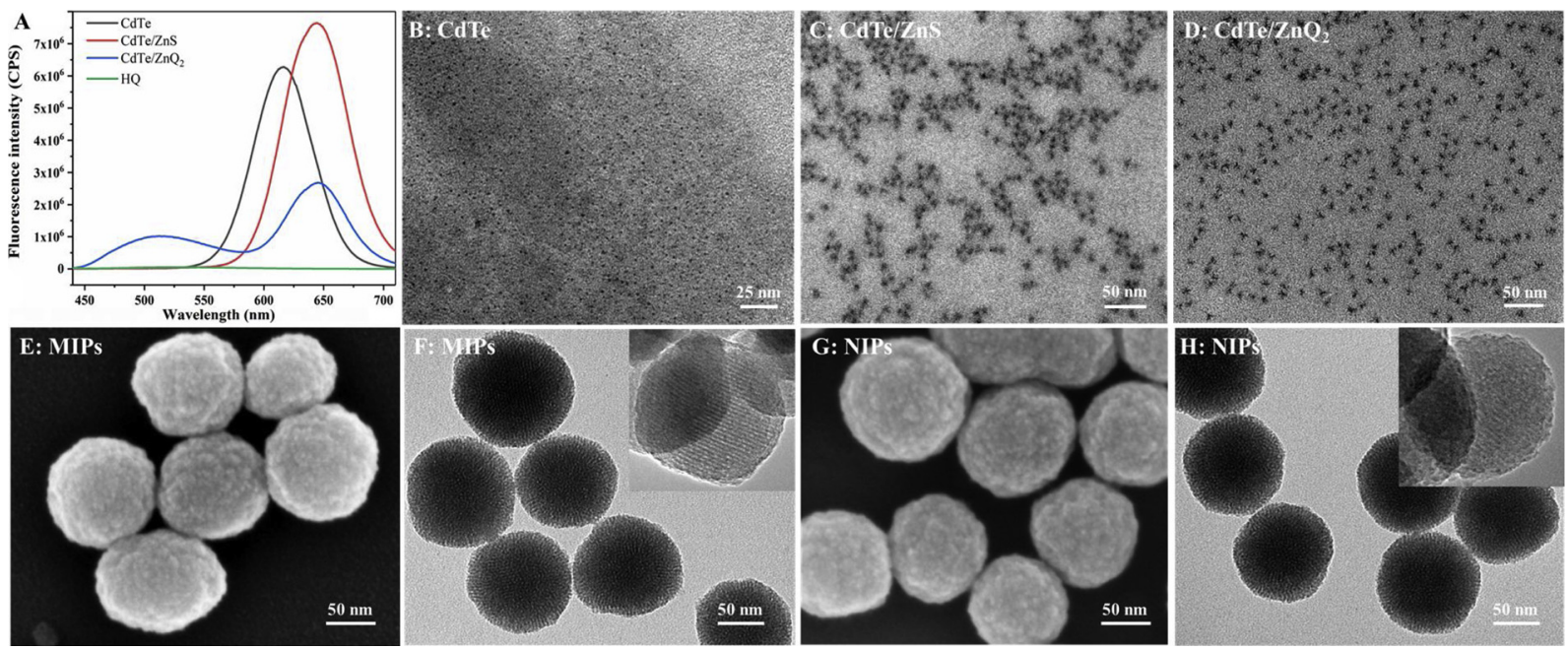

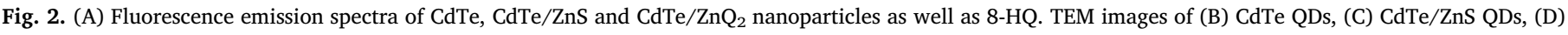

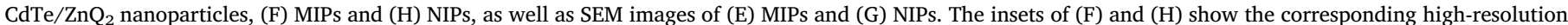
TEM images.

capping reagent and sulfur source. The anchored ZnS shell played the three roles of decreasing toxicity, enlarging CdTe emission wavelength and providing $\mathrm{Zn}^{2+}$ for chelation reaction. Specifically, compared with the incipient CdTe QDs, the synthesized CdTe/ZnS QDs had less toxicity and better biocompatibility since the dissociation of $\mathrm{Cd}^{2+}$ had been suppressed by ZnS shell. Simultaneously, the ZnS shell efficiently eliminated the surface dangling bonds and defects, so that the fluorescence intensity was enhanced and the fluorescence emission had a bathochromic shift from 616 to $643 \mathrm{~nm}$, leaving room for blue shift to specific wavelength for overlapping the absorption spectrum of Brilliant Blue. Subsequently, non-fluorescent HQ was chelated with the surface $\mathrm{Zn}^{2+}$ ions to obtain fluorescent HQ-metal complex namely $\mathrm{ZnQ}_{2}$, which was further anchored on the surface of CdTe/ZnS QDs via the dangling sulfide bond. Thereupon, the attached complex led to the formation of a single-component but dual-emission $\mathrm{CdTe} / \mathrm{ZnQ}_{2}$ nanoparticles, with an emission peak at $510 \mathrm{~nm}$ from $\mathrm{ZnQ}_{2}$ complex and the other peak at $630 \mathrm{~nm}$ from CdTe core. The dual emission was derived from a single type of nanoparticles rather than a simple mixture of $\mathrm{CdTe} / \mathrm{ZnS}$ and $\mathrm{HQ}$, because HQ in aqueous solution was non-fluorescent while emission at $510 \mathrm{~nm}$ appeared after chelation with CdTe/ZnS QDs (Fig. 2A). TEM images in Fig. 2B-D suggests that CdTe, CdTe/ZnS and CdTe/ZnQ 2 nanoparticles possessed good dispersed crystalline structures, and had the average diameter of approximate 2,5 and $6 \mathrm{~nm}$, respectively, where $\mathrm{CdTe} / \mathrm{ZnS}$ shared the similar morphology with previous study [15].

Based on the synthesis of bicolor-emitting $\mathrm{CdTe} / \mathrm{ZnQ}_{2}$ nanoparticles, the MIPs sensor was prepared by one-pot condensation of TEOS in the presence of pre-polymerized APTES-Brilliant Blue complex and $\mathrm{CTAB}$ as soft template for mesoporous structure, with subsequent templates removal of Brilliant Blue and CTAB. Hence, the resulting recognition sites can be complementary to Brilliant Blue in shape, size and functional groups, in favor of the subsequent rebinding of them via hydrogen bonds and inducing changes in the fluorescence characteristics. Mesoporous structured imprinting matrix was adopted for the reason that, compared with the highly cross-linked sphere or irregular bulk MIPs, the mesoporous one possesses numerous uniform mesoporous and higher specific surface area that can effectively reduce mass transfer resistance and make recognition site more accessible to Brilliant Blue. What's more, this single-component bicolor-emitting $\mathrm{CdTe} / \mathrm{ZnQ}_{2}$ based sensor avoided the tedious chemical coupling and simplified the construction process, especially compared with the traditional ratiometric fluorescence sensors prepared by combining two individual fluorescence substances whose preparation is tediously multi-step with sophisticated coupling or chemical modification [14].
The morphologies of MIPs sensor and the corresponding NIPs were characterized by SEM/TEM/HRTEM in Fig. 2E-H, respectively. As seen, the monodispersed MIPs and NIPs shared similar average size of approximate $120 \mathrm{~nm}$, as well as the analogous morphology of rough surface and hexagonal tunnels channel, which suggested the existence of mesoporous structure in the imprinting matrix. Elemental mapping of the prepared dual-emission MIPs and NIPs were also observed (Fig. S2). Clearly, the existence of various elements of C, N, Si, O, Cd, Te, Zn and S suggested the successful construction of $\mathrm{SiO}_{2}$ matrices and introduction of dual-emission $\mathrm{CdTe} / \mathrm{ZnQ}_{2}$ nanoparticles and functional groups from APTES. As control, only $\mathrm{C}, \mathrm{N}, \mathrm{Si}$ and $\mathrm{O}$ were observed for the nonfluorescent MIPs prepared without $\mathrm{CdTe} / \mathrm{ZnQ}_{2}$.

Fig. S3 displays the FT-IR spectra of MIPs before and after elution and NIPs. Comparing the FT-IR spectra of MIPs before and after elution, the typical absorption bands of CTAB in region of $3000-2800 \mathrm{~cm}^{-1}$ (vibrations of $-\mathrm{CH}_{2}-$ ) and peaks of Brilliant Blue ranging from $1650-1450 \mathrm{~cm}^{-1}$ (stretching vibration of $\mathrm{C}=\mathrm{C}$ in benzene rings) were greatly weakened, indicating the successful removal of CTAB and Brilliant Blue. Besides, no obvious difference was observed in major backbones between MIPs and NIPs: the characteristic peaks around 461 and $794 \mathrm{~cm}^{-1}$ could be ascribed to $\mathrm{Si}-\mathrm{O}$ stretching vibration and the wide strong band in $1065 \mathrm{~cm}^{-1}$ belonged to $\mathrm{Si}-\mathrm{O}-\mathrm{Si}$ stretching vibration, illustrating the presence of $\mathrm{SiO}_{2}$ matrices in MIPs and NIPs; the stretching vibration of $\mathrm{N}-\mathrm{H}$ at $3428 \mathrm{~cm}^{-1}$ proved the amino group was modified into the imprinting matrix to act as the functional group. Obviously, MIPs and NIPs shared the similar primary compositions. Consequently, mesoporous structured MIPs were obtained.

\subsection{Possible detection principle of the MIPs sensor toward Brilliant Blue}

Before Brilliant Blue recognition, the MIPs sensor displayed two well-resolved emission peaks at 510 and $630 \mathrm{~nm}$ under a single wavelength excitation at $365 \mathrm{~nm}$, and emitted fluorescence in orange window under a $365 \mathrm{~nm}$ UV lamp. Depending on the memory of imprinted sites, the sensor recognized and rebound Brilliant Blue via hydrogen bonds forming between the amino group of APTES and the sulfonic group of Brilliant Blue (Fig. 1). The binding events were transduced into fluorescence signals that the fluorescence at $630 \mathrm{~nm}$ of CdTe segment was quenched, whereas the fluorescence of $\mathrm{ZnQ}_{2}$ at $510 \mathrm{~nm}$ was inert with intensity scarcely changing. The CdTe and $\mathrm{ZnQ}_{2}$ provided reliable target-dependent response function and internal reference, respectively. Upon the change in $I_{630} / I_{510}$, the color of the sensor gradually varied from orange to green and thus facilitated the 
A

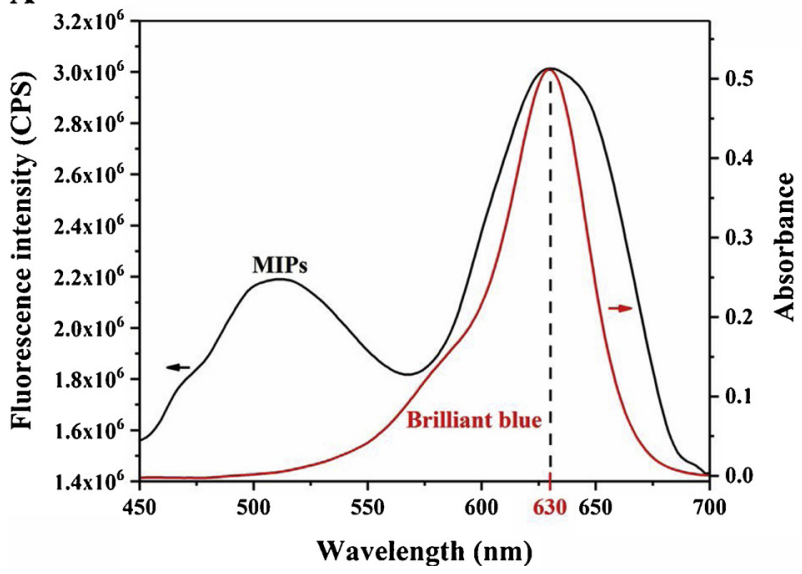

B

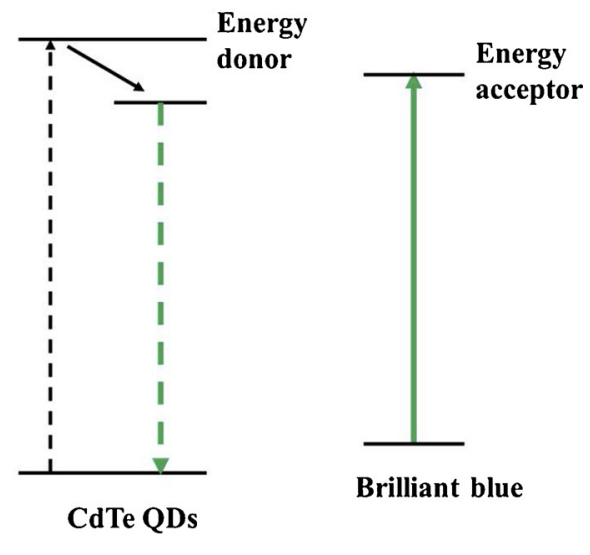

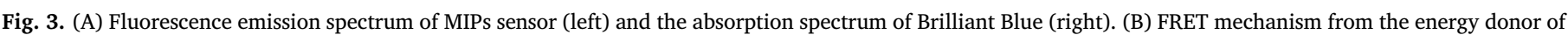

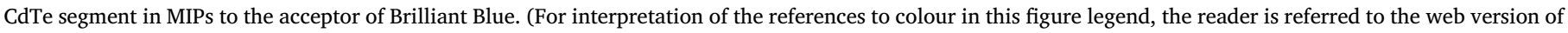
this article.)

visualization of Brilliant Blue.

The FRET mechanism was reasonably speculated to answer for the fluorescence quenching, owing to the overlap between the emission spectrum of CdTe segment (energy donor) and the absorption spectrum of Brilliant Blue (energy acceptor), as shown in Fig. 3A. When they were spatially close to each other, the energy from the pathway where the excited-state electrons of the energy donor returned to the ground state could transfer to Brilliant Blue and further transit the ground-state electrons to the excited state, due to the polar-polar resonance of donor and acceptor (Fig. 3B). Therefore, highly efficient fluorescence quenching of donor occurred [28]. Moreover, it is noteworthy that the overlapped degree of the energy donor and acceptor had a significant impact on the FRET. So, we adjusted the emission spectrum of donor to center at suitable wavelength of $630 \mathrm{~nm}$, ensuring the overlap at the maximum extent.

\subsection{Condition optimization}

Various parameters affecting the sensing performance were systematically optimized, including the emission position of CdTe segment, the intensity ratio of two emission peaks in MIPs sensor, as well as the dosage of MIPs, $\mathrm{pH}$ and response time for the fluorescence measurement, using the quenching amount of $\left(I_{630} / I_{510}\right)_{0} /\left(I_{630} / I_{510}\right)$ as the index.

A proper emission position of CdTe QDs could guarantee the largest overlap with the absorption band of Brilliant Blue (centered at $630 \mathrm{~nm}$ ) to positively affect the energy transfer procedure. Experimentally, the fluorescence peak of CdTe segment in MIPs sensor mainly depended on its original emission wavelength, the reaction time for preparing CdTe/ ZnS QDs and the doping amount of HQ. In our study, the fluorescence color of CdTe QDs turned from green to orange quickly and further became red in an incredibly slow step, and hard to attain $630 \mathrm{~nm}$ even spending more than 2 days with fluorescence quantum yield largely decreased. To avoid the long-term preparation and the depressing of the fluorescence quantum yield, we prepared the CdTe QDs centered at $616 \mathrm{~nm}$ and further anchored the $\mathrm{ZnS}$ shell onto them. As shown in Fig. S4, the emission wavelength gradually shifted from $616 \mathrm{~nm}$ to a longer wavelength with the time increase for $\mathrm{ZnS}$ shell growth, and reached $643 \mathrm{~nm}$ after $40 \mathrm{~min}$ with higher fluorescence quantum yield because of the surface passivation effect. Furthermore, gradual blue shift was observed upon increasing doping amount of HQ (Fig. S5). Hence, the ZnS shell growth time was set to be $40 \mathrm{~min}$ to make CdTe emit at $643 \mathrm{~nm}$, longer than $630 \mathrm{~nm}$ to leave room for blue shift caused by HQ chelation. Obviously, the emission of CdTe can be flexibly controlled to a suitable position for sensing other colored templates besides Brilliant Blue.
The fluorescence intensity ratio of two emission peaks can affect the ratiometric detection [13]. As we know, the doping dosage of HQ not only affected the blue shift of CdTe segment, but also caused fluorescence appearing of $\mathrm{ZnQ}_{2}$ and fluorescence quenching of CdTe to finally determine the intensity ratio. Fig. 66 shows the fluorescence spectra (A) of MIPs with different peak intensity ratios (B) prepared by different dosage of HQ. As seen, only the CdTe peak was observed when HQ wasn't added, while a bit more HQ produced an equal intensity between CdTe and $\mathrm{ZnQ}_{2}$ peaks. This was because the increasing HQ resulted in increasing $\mathrm{ZnQ}_{2}$ complex generation and occupation to the surface of $\mathrm{CdTe} / \mathrm{ZnS}$, and the charge recombination of electron transfer from the photoexcited CdTe QDs to the coordinated $\mathrm{ZnQ}_{2}$ caused fluorescence quenching of CdTe [15]. Hence, the relative intensity of the peaks could be tuned by changing the concentrations of the HQ ligands. Ultra-weak intensity of reference $\mathrm{ZnQ}_{2}$ would be masked by CdTe, so that the ratiometric fluorescence performed no practical function and only narrow color variation could be observed. However, MIPs sensor with equal intensity in $\mathrm{ZnQ}_{2}$ and CdTe could emit yellow under a UV lamp and change to be green when CdTe was quenched, lacking the orange part. What's worse, the relatively low intensity of CdTe could lead to low sensitivity upon target rebinding. Hence, $50 \mu \mathrm{L}$ of $\mathrm{HQ}$ at $15 \mathrm{mmol} \cdot \mathrm{L}^{-1}$ was used for $\mathrm{CdTe} / \mathrm{ZnQ}_{2}$ preparation, providing the maximum color change range from orange to green.

The amount of MIPs in the sensing system would play an important role in Brilliant Blue measurement. As seen from Fig. S7, the fluorescence intensities at 510 and $630 \mathrm{~nm}$ both increased with the increasing amount of MIPs from 292 to $730 \mu \mathrm{g} \cdot \mathrm{mL}^{-1}$. Addition of $1 \mu \mathrm{mol} \cdot \mathrm{L}^{-1}$ Brilliant Blue caused fluorescence quenched at $630 \mathrm{~nm}$, while no obvious change was observed at $510 \mathrm{~nm}$ no matter how many MIPs were added. However, MIPs at $583 \mu \mathrm{g} \cdot \mathrm{mL}^{-1}$ caused the biggest quenching amount of $\left(I_{630} / I_{510}\right)_{0} /\left(I_{630} / I_{510}\right)$ approximately 1.384 . Actually, excessively high amounts of MIPs resulted in low sensitivity, whereas excessively low amounts of MIPs led to narrow detection range. Hence, $583 \mu \mathrm{g} \cdot \mathrm{mL}^{-1}$ was adopted as the amount of MIPs.

The influence of $\mathrm{pH}$ on the ratiometric detection was also investigated. As seen from Fig. S8, with the enhancement of alkalinity, the values of $\left(I_{630} / I_{510}\right)_{0} /\left(I_{630} / I_{510}\right)$ toward Brilliant Blue regularly increased until $\mathrm{pH} 9.0$ and then almost unchanged. This was in accord with the accepted theory that the consistency of $\mathrm{pH}$ in imprinting system (alkaline solution in this method) and sensing system could avoid the conformation change of the recognition sites, and thereby attain its specificity and the best recognition performance [29,30]. In addition, $\mathrm{pH}$ affected the emission of the QDs, and the fluorescence emission intensity was high under alkaline conditions. Both high-efficiency rebinding of Brilliant Blue and the great emission efficiency of 

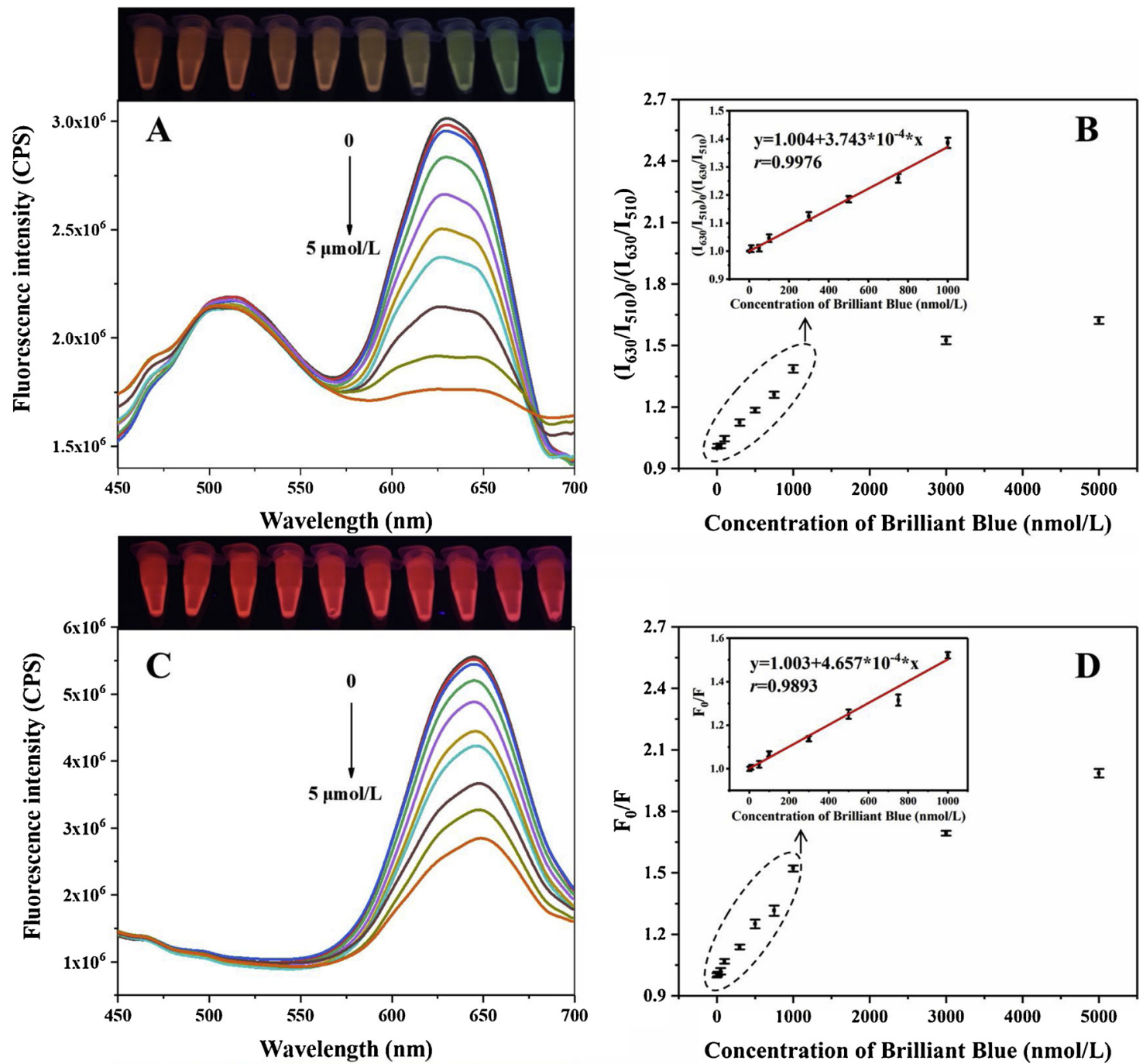

Concentration of Brilliant Blue (nmol/L)
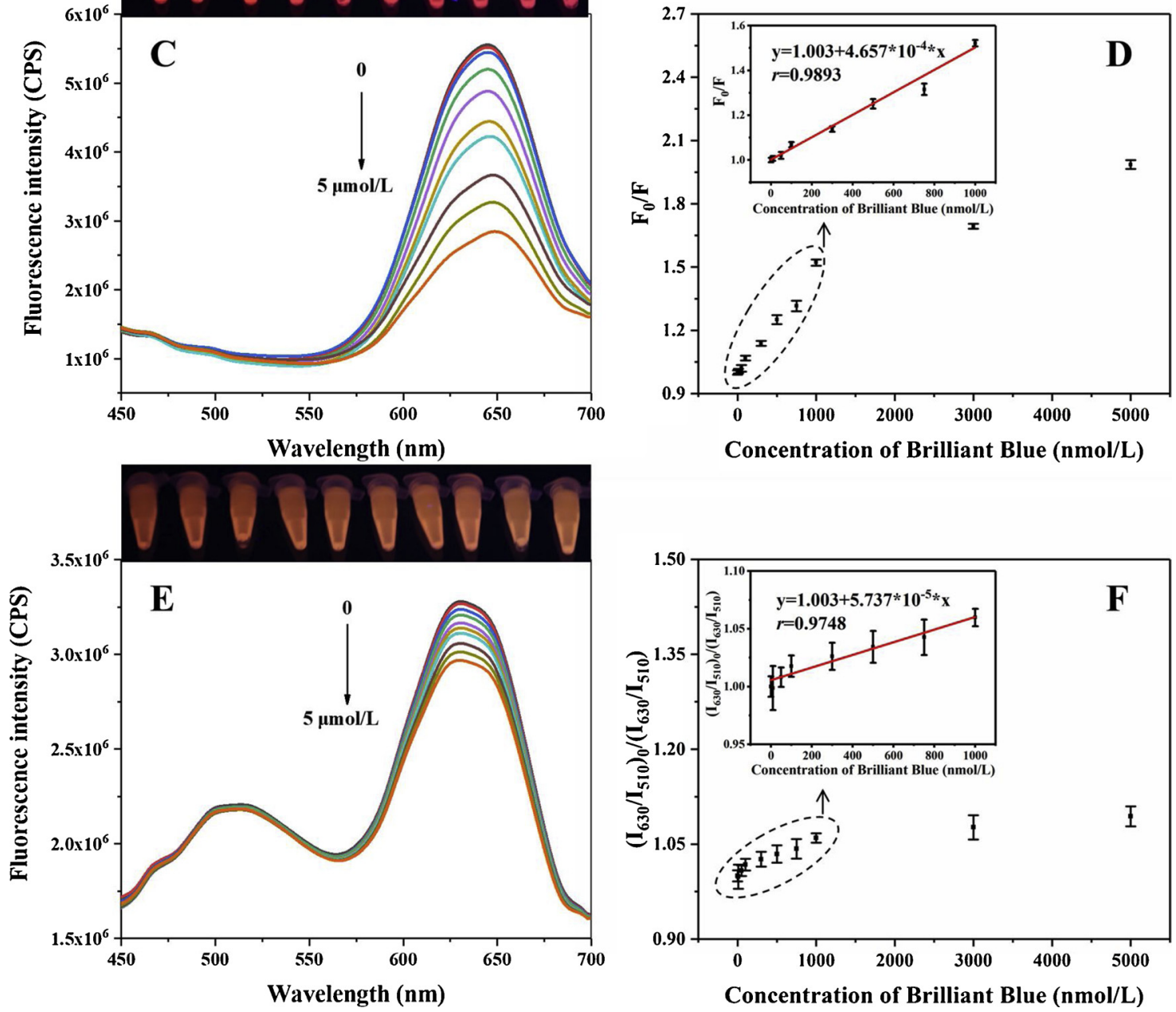

Fig. 4. Fluorescence spectra and the corresponding quenching amount $\left(I_{630} / I_{510}\right)_{0} /\left(I_{630} / I_{510}\right)$ with Brilliant Blue concentration from 0 to $5 \mu$ mol-L $\mathrm{L}^{-1}$ for $(\mathrm{A}$, B) dualemission MIPs sensor of mMIP@CdTe/ZnQ 2 , (C, D) single-emission MIPs sensor of mMIP@CdTe/ZnS and (E, F) dual-emission NIPs sensor. The insets of (B, D, F) show the linear fitting of quenching amount $\left(I_{630} / I_{510}\right)_{0} /\left(I_{630} / I_{510}\right)$ with Brilliant Blue concentration from 0 to $1 \mu$ mol: $\mathrm{L}^{-1}$. The photography images in the upper $(\mathrm{A}$, C, E) from left to right are their corresponding fluorescence color evolution taken under a $365 \mathrm{~nm}$ UV lamp. (For interpretation of the references to colour in this figure legend, the reader is referred to the web version of this article.)

QDs would improve the sensing sensitivity. Hence, $\mathrm{pH} 9.0$ was adopted for the further study.

The response time for MIPs sensor to reach equilibrium is one important parameter to assess the accessibility of recognition sites. The fluorescence intensity ratio of $I_{630} / I_{510}$ had a cliff-type fall after adding Brilliant Blue within $2 \mathrm{~min}$, and reached equilibrium within $6 \mathrm{~min}$ (Fig. 


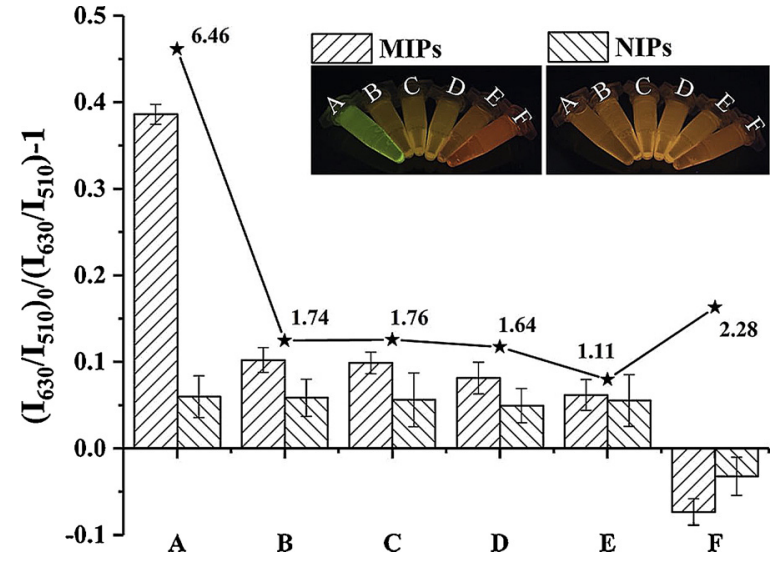

Fig. 5. Selectivity of MIPs and NIPs for (A) Brilliant Blue over other food colorants including (B) Gardenia Blue, (C) Indigotine, (D) Spirulina Blue, (E) Sodium Copper Chlorophyllin and (F) Sunset Yellow at the same concentration of $1 \mu \mathrm{mol} \cdot \mathrm{L}^{-1}$. The five-pointed stars stand for the imprinting effect between MIPs and NIPs. Inset: the photography images are their corresponding fluorescence color of MIPs and NIPs after recognition. (For interpretation of the references to colour in this figure legend, the reader is referred to the web version of this article.)

S9). The fast response rate verified the low mass transfer resistance and high site accessibility of mesoporous structure, since there were a number of interconnected mesoporous and all recognition sites were located in the ultrathin imprinting matrix among the mesoporous. Therefore, the response time was fixed at $6 \mathrm{~min}$.

\subsection{Analytical performance of MIPs sensor}

Under the optimized conditions, the fluorescence spectra of the MIPs sensor after rebinding targeted Brilliant Blue of different concentrations were recorded, for evaluating its quantitative analysis performance. As shown in Fig. 4A, in the absence of Brilliant Blue, two well-resolved emission peaks at 510 and $630 \mathrm{~nm}$ were displayed; with Brilliant Blue adding from 0 to $5 \mu \mathrm{mol} \cdot \mathrm{L}^{-1}$, the fluorescence intensity at $630 \mathrm{~nm}$ was regularly decreased, while the intensity at $510 \mathrm{~nm}$ had no obvious change. The ratio of the fluorescence intensity of $\left(I_{630} / I_{510}\right)_{0} /$ $\left(I_{630} / I_{510}\right)$, standing for the quenching level against the original ones, was linearly related to the concentration of Brilliant Blue. As shown in Fig. 4B, the slope of quenching amount versus template concentration from 0 to $1 \mu \mathrm{mol} \cdot \mathrm{L}^{-1}$ was higher than that from 1 to $5 \mu \mathrm{mol} \cdot \mathrm{L}^{-1}$, i.e., higher sensitivity were achieved in lower concentration. This was because imprinted cavities suited at the surface or in the proximity of the surface provided higher accessibility and lower mass transfer resistance, while lower sensitivity was toward higher concentration Brilliant Blue when a number of sites had been occupied. As seen from Fig. 4B, quite low relative standard deviations (RSD, $n=3$ ) of $1.1-2.3 \%$ were obtained, demonstrating the reliability of the ratiometric fluorescence detection for quantitative analysis. And based on $3 \sigma / s$, where $\sigma$ means the standard deviation of the blank measurements and $s$ means the slope of calibration curve, the limit of detection (LOD) was attained down to $8.8 \mathrm{nmol} \cdot \mathrm{L}^{-1}$. The value is much lower than the permitted maximum level in foodstuffs. As for the foods where Brilliant Blue is not allowed, the LOD value is promising to judge the presence or absence of Brilliant Blue. As shown in the upper of Fig. 4A, continuous and distinguishable fluorescence color changes from orange to yellowish orange to yellowish green to green also appeared with the addition of Brilliant Blue, by virtue of the regular quenching of red fluorescence but no change in green one, making the visual detection of Brilliant Blue feasible by the naked eye.

The performance of the ratiometric MIPs sensor was compared with that of single-emission MIPs sensor, where no HQ was doped and only
CdTe segment emitted fluorescence. As shown in Fig. 4C, after rebinding Brilliant Blue, similar quenching trend of CdTe QDs was attained; however, as shown in Fig. $4 \mathrm{D}$, the linear relation between the quenching amount and concentration of Brilliant Blue was not as good as that of ratiometric MIPs (Fig. 4B) with a larger correlation coefficient $(r)$. This was possibly because that the fluorescence spectra were compromised by a variety of analyte-independent factors and the reference $\mathrm{ZnQ}_{2}$ in the ratiometric sensor served the self-correction effect to enhance the reliability in Brilliant Blue detection. What's more, the color variation of the single-emission MIPs after Brilliant Blue recognition was hard to observe because only the brightness changed (in the upper of Fig. 4C).

The existence of recognition sites for Brilliant Blue was proved by sensing performance comparison with NIPs. As shown in Fig. 4E and F, only slight quenching at $630 \mathrm{~nm}$ and the consequent smoother slopes were observed, and meanwhile, the fluorescence color change was hard to be distinguished (in the upper of Fig. 4E). Imprinting factor (IF), defined as the ratio of slopes of the standard curves, i.e. $K_{\mathrm{MIP}}$ to $K_{\mathrm{NIP}}$, was used to evaluate the imprinting effect of MIPs; and the IF values were calculated as 7.1 for $0-1 \mu \mathrm{mol} \cdot \mathrm{L}^{-1}$ concentration range. Hence, it could be reasonably concluded that no complementary recognition sites were present towards Brilliant Blue and only nonspecific binding located on the surface occurred in NIPs. So, the MIPs based ratiometric fluorescence sensor was capable to sensitively detect Brilliant Blue.

Furthermore, the selectivity of MIPs was evaluated by comparing the fluorescence emission ratio changes toward other food colorants at the same concentration as that of Brilliant Blue. As seen from Fig. 5, compared with the remarkable ratio and color changes towards Brilliant Blue (histogram A), other tested blue colorants of Gardenia Blue, Indigotine and Spirulina Blue all caused low variance (histogram B-D, respectively), owing to the significant difference in molecular structure from the template molecule. The green colorant of Sodium Copper Chlorophyllin made less change (histogram E) due to different structure and no overlap between its absorption spectrum and the emission spectrum of MIPs. As for the yellow colorant of Sunset Yellow, its absorption spectrum overlapped the emission peak of $\mathrm{ZnQ}_{2}$ complex and therefore caused its quenching but no change in CdTe segment; however, no significant influence (histogram F) was made because of no complementary recognition sites in MIPs. On the other hand, similar inconspicuous fluorescence emission and color changes were observed in NIPs for Brilliant Blue and other colorants owing to the lack of recognition sites. Excitingly, once rebinding Brilliant Blue, the MIPs changed from original orange to yellowish green; this remarkable color change demonstrated the visual precedence over other food colorants at the same concentration (in the inset of Fig. 5). Hence, the MIPs sensor had high selectivity toward the template molecule of Brilliant Blue for the readily visual detection.

Additionally, the stability of the MIPs sensor was investigated by repeatedly measuring the fluorescence intensity before and after Brilliant Blue detection every day. As displayed in Fig. S10, within 10 days, the fluorescence ratio of $I_{630} / I_{510}$ almost unchanged, with the deviation less than $3 \%$ of its original value measured at the first day, and the recognition ability toward Brilliant Blue was also kept constant without obvious change. These results implied the good physical and chemical stability due to the excellent protection of MIPs coating as well as the self-correction effect. Consequently, the MIPs had demonstrated excellent sensing performances of high sensitivity, precision, selectivity and stability together with ideal visual ability.

\subsection{Application of the sensor to real food samples}

To explore the applicability of the ratiometric fluorescence MIPs sensor in real sample analysis, six typical food samples of carbonated beverage, brandy cocktail, popping candy, chocolate candy, mung bean cake and dried blueberry were investigated by our fluorescence sensor and the traditional methods of HPLC-UV and UV-vis. All the 
Table 1

Determination of Brilliant Blue in six typical food samples by the present MIPs sensor and conventional HPLC-UV and. UV-vis.

\begin{tabular}{llll}
\hline Food Samples & \multicolumn{2}{l}{ Detected Brilliant Blue $\left((\mathrm{mg} / \mathrm{kg})^{\mathrm{a}} \pm \mathrm{RSD}^{\mathrm{b}}(\%)\right)$} \\
\hline & Present Sensor & HPLC-UV & UV-vis \\
Carbonated Beverage $^{\mathrm{c}}$ & $0.409 \pm 1.985$ & $0.390 \pm 2.860$ & $0.389 \pm 2.182$ \\
Brandy Cocktail $^{\mathrm{c}}$ & $1.806 \pm 3.270$ & $1.885 \pm 4.356$ & $1.938 \pm 3.605$ \\
Popping Candy $^{\mathrm{d}}$ & $3.219 \pm 2.101$ & $3.400 \pm 2.906$ & $3.474 \pm 2.410$ \\
Chocolate Candy $^{\mathrm{d}}$ & $41.031 \pm 3.457$ & $39.666 \pm 1.984$ & $41.191 \pm 2.745$ \\
Mung Bean Cake $^{\mathrm{e}}$ & $0.206 \pm 3.097$ & $0.191 \pm 3.923$ & $0.204 \pm 3.268$ \\
Dried Blueberry $^{\mathrm{e}}$ & $0.354 \pm 3.447$ & $0.327 \pm 3.782$ & $2.323 \pm 2.515$ \\
\end{tabular}

a Average value from three parallel determinations.

b Relative standard deviation $(n=3)$.

c The authorized maximum level is $25 \mathrm{mg} / \mathrm{kg}$.

d The authorized maximum level is $300 \mathrm{mg} / \mathrm{kg}$.

e Not allowed to add synthesis colorants.

experimental results were listed in Table 1 and schematically shown in Fig. S10, respectively. As seen, by using our sensor, the detected values for Brilliant Blue in carbonated beverage and brandy cocktail were 0.409 and $1.701 \mathrm{mg} / \mathrm{kg}$, respectively, much lower than the authorized maximal level of $25 \mathrm{mg} / \mathrm{kg}$; the detected Brilliant Blue in popping candy and chocolate candy was 3.219 and $41.031 \mathrm{mg} / \mathrm{kg}$, respectively, much lower than the restricted maximal level of $300 \mathrm{mg} / \mathrm{kg}$. Although mung bean cake and dried blueberry are not allowed to add synthesis colorants, Brilliant Blue of 0.206 and $0.354 \mathrm{mg} / \mathrm{kg}$ was still found, respectively. The low RSD values in a range of $1.870-3.457 \%$ suggested the present sensor was highly accurate for quantitative determination. All the above judgment of Brilliant Blue addition refers to RPC National Standard GB 2760-2014 (Table S1).

In addition, according to the observation values for all the tested samples, the results by our sensor were very consistent with those obtained from HPLC-UV (Table 1 and Fig. S11). The $t$-test confirmed that there was no significant difference between the developed sensor and the HPLC-UV determination at 95\% confidence level. As well as, for the former five samples, the measured Brilliant Blue by our sensing method was comparable with that attained by UV-vis. Hence, the sensing method was feasible for real sample analysis with a high accuracy and good reliability. It should be noted that an abnormally high value $(2.323 \mathrm{mg} / \mathrm{kg})$ occurred in dried blueberry by UV-vis determination, showing ultra-high deviation of $632 \%$ from HPLC-UV method, possibly owing to the existence of other blue colorants with absorption spectra covering that of Brilliant Blue. On the other hand, the abnormal results by UV-vis could further indicate that our fluorescence sensor was practically applicable, accurate and reliable for the direct detection of Brilliant Blue in real samples. Moreover, our presented MIPs sensor method is of convenience for visually detecting Brilliant Blue via the fluorescence color change, which allowed untrained people to readily perform analysis but without learn about the sophisticated sensing principle involved.

\section{Conclusions}

To sum up, a mesoporous structured molecular imprinting ratiometric fluorescence sensor was constructed via the dual-emission colorcontrollable $\mathrm{CdTe} / \mathrm{ZnQ}_{2}$ nanoparticles. Based on the FRET mechanism, the sensor selectively recognized and sensitively quantified the trace Brilliant Blue in complicated food samples, accompanying with widerange fluorescence color variation for visual detection. This one-pot construction strategy depending on the dual-emission nanoparticles effectively avoided tedious multistep preparation and sophisticated coupling or chemical modification process, and thereby could provide a new strategy for imprinting ratiometric fluorescence sensor construction. Besides, by virtue of the Brilliant Blue imprinting, an analytical model of proof-of-concept, it is potentially promising to develop a general applicable recognition and detection platform for colored substances based on FRET, by adjusting the emission peak (e.g., of CdTe segment) at a suitable emission wavelength. Therefore, we reasonably believe that this work can promote the smart construction and field application of novel molecular imprinting ratiometric fluorescence sensors.

\section{Conflict of interest}

The authors declare no competing financial interest.

\section{Acknowledgments}

This work was financially supported by the National Natural Science Foundation of China (21667018, 21804010, 21876199, 31660482, 31860470, 41776110, 21575159), the Natural Science Foundation of Shandong Province of China (ZR2016BL25), and the Department of Science and Technology of Shandong Province of China (GG201709290055).

\section{Appendix A. Supplementary data}

Supplementary material related to this article can be found, in the online version, at doi:https://doi.org/10.1016/j.snb.2018.12.134.

\section{References}

[1] L. Chen, S. Xu, J. Li, Recent advances in molecular imprinting technology: current status, challenges and highlighted applications, Chem. Soc. Rev. 40 (2011) 2922-2942.

[2] J. Luan, T. Xu, J. Cashin, J.J. Morrissey, E.D. Kharasch, S. Singamaneni, Environmental stability of plasmonic biosensors based on natural vs. artificial antibody, Anal. Chem. 90 (2018) 7880-7887.

[3] L. Chen, X. Wang, W. Lu, X. Wu, J. Li, Molecular imprinting: perspectives and applications, Chem. Soc. Rev. 45 (2016) 2137-2211.

[4] Q. Yang, J. Li, X. Wang, H. Peng, H. Xiong, L. Chen, Strategies of molecular imprinting-based fluorescence sensors for chemical and biological analysis, Biosens. Bioelectron. 112 (2018) 54-71.

[5] Z. Zhou, T. Li, W. Xu, W. Huang, N. Wang, W. Yang, Synthesis and characterization of fluorescence molecularly imprinted polymers as sensor for highly sensitive detection of dibutyl phthalate from tap water samples, Sens. Actuators B Chem. 240 (2017) 1114-1122.

[6] J. Zhou, Y. Yang, C. Zhang, Toward biocompatible semiconductor quantum dots: from biosynthesis and bioconjugation to biomedical application, Chem. Rev. 115 (2015) 11669-11717.

[7] A.M. Derfus, W. Chan, S.N. Bhatia, Probing the cytotoxicity of semiconductor quantum dots, Nano Lett. 4 (2004) 11-18.

[8] Y. Liu, J. Yu, In situ synthesis of highly luminescent glutathione-capped CdTe/ZnS quantum dots with biocompatibility, J. Colloid Interf. Sci. 351 (2010) 1-9.

[9] W. Li, H. Zhang, S. Chen, Y. Liu, J. Zhuang, B. Lei, Synthesis of molecularly imprinted carbon dot grafted $\mathrm{YVO} 4: \mathrm{Eu}^{3+}$ for the ratiometric fluorescent determination of paranitrophenol, Biosens. Bioelectron. 86 (2016) 706-713.

[10] X. Wang, J. Yu, X. Wu, J. Fu, Q. Kang, D. Shen, J. Li, L. Chen, A molecular imprinting-based turn-on ratiometric fluorescence sensor for highly selective and sensitive detection of 2,4-dichlorophenoxyacetic acid (2,4-D), Biosens. Bioelectron. 81 (2016) 438-444.

[11] K. Zhang, H. Zhou, Q. Mei, S. Wang, G. Guan, R. Liu, J. Zhang, Z. Zhang, Instant visual detection of trinitrotoluene particulates on various surfaces by ratiometric fluorescence of dual-emission quantum dots hybrid, J. Am. Chem. Soc. 133 (2011) 8424-8427.

[12] S. Xu, H. Lu, Ratiometric fluorescence and mesoporous structure dual signal amplification for sensitive and selective detection of TNT based on MIP@QD fluorescence sensors, Chem. Commun. (Camb.) 51 (2015) 3200-3203.

[13] S. Xu, H. Lu, One-pot synthesis of mesoporous structured ratiometric fluorescence molecularly imprinted sensor for highly sensitive detection of melamine from milk samples, Biosens. Bioelectron. 73 (2015) 160-166.

[14] H. Lu, S. Xu, Visualizing BPA by molecularly imprinted ratiometric fluorescence sensor based on dual emission nanoparticles, Biosens. Bioelectron. 92 (2017) $147-153$.

[15] B. Liu, C. Tong, L. Feng, C. Wang, Y. He, C. Lu, Water-soluble polymer functionalized CdTe/ZnS quantum dots: a facile ratiometric fluorescent probe for sensitive and selective detection of nitroaromatic explosives, Chem. Eur. J. 20 (2014) 2132-2137.

[16] N. Martins, C.L. Roriz, P. Morales, L. Barros, I.C.F.R. Ferreira, Food colorants: challenges, opportunities and current desires of agro-industries to ensure consumer expectations and regulatory practices, Trends Food Sci. Tech. 52 (2016) 1-15.

[17] Y. Ou, X. Wang, K. Lai, Y. Huang, B.A. Rasco, Y. Fan, Gold nanorods as surface- 
enhanced Raman spectroscopy substrates for rapid and sensitive analysis of allura red and sunset yellow in beverages, J. Agric. Food Chem. 66 (2018) 2954-2961.

[18] P. Amchova, H. Kotolova, J. Ruda-Kucerova, Health safety issues of synthetic food colorants, Regul. Toxicol. Pharmacol. 73 (2015) 914-922.

[19] D. McCann, A. Barrett, A. Cooper, D. Crumpler, L. Dalen, K. Grimshaw, E. Kitchin, K. Lok, L. Porteous, E. Prince, E.J.S. Sonuga-Barke, J.O. Warner, J. Stevenson, Food additives and hyperactive behaviour in 3-year-old and 8/9-year-old children in the community: a randomised, double-blinded, placebo-controlled trial, Lancet 370 (2007) 1560-1567.

[20] EFSA Panel on Food Additives and Nutrient Sources added to Food (ANS), Scientific Opinion on the reevaluation of Brilliant Blue FCF (E 133) as a food additive, Efsa J. 8 (11) (2010) 1853, https://doi.org/10.2903/j.efsa.2010.1853 Available online www.efsa.europa.eu.

[21] T.M. Coelho, E.C. Vidotti, M.C. Rollemberg, A.N. Medina, M.L. Baesso, N. Cella, A.C. Bento, Photoacoustic spectroscopy as a tool for determination of food dyes: comparison with first derivative spectrophotometry, Talanta 81 (2010) 202-207.

[22] F. Liu, C. Liu, W. Li, A. Tang, Dispersive solid-phase microextraction and capillary electrophoresis separation of food colorants in beverages using diamino moiety functionalized silica nanoparticles as both extractant and pseudostationary phase, Talanta 132 (2015) 366-372.

[23] P. Sierra-Rosales, C. Toledo-Neira, J.A. Squella, Electrochemical determination of food colorants in soft drinks using MWCNT-modified GCEs, Sens. Actuators B Chem 240 (2017) 1257-1264.

[24] O. Sha, X. Zhu, Y. Feng, W. Ma, Aqueous two-phase based on ionic liquid liquidliquid microextraction for simultaneous determination of five synthetic food colourants in different food samples by high-performance liquid chromatography, Food Chem. 174 (2015) 380-386.

[25] V. Peksa, M. Jahn, L. Stolcova, V. Schulz, J. Proska, M. Prochazka, K. Weber, D. Cialla-May, J. Popp, Quantitative SERS analysis of azorubine (E 122) in sweet drinks, Anal. Chem. 87 (2015) 2840-2844.

[26] S. Xu, H. Lu, J. Li, X. Song, A. Wang, L. Chen, S. Han, Dummy molecularly imprinted polymers-capped CdTe quantum dots for the fluorescent sensing of 2,4,6-trinitrotoluene, ACS Appl. Mater. Interfaces 5 (2013) 8146-8154.

[27] S. Pramanik, S. Bhandari, S. Roy, A. Chattopadhyay, Synchronous tricolor emissionbased white light from quantum dot complex, J. Phys. Chem. Lett. 6 (2015) 1270-1274.

[28] X. Wang, J. Yu, Q. Kang, D. Shen, J. Li, L. Chen, Molecular imprinting ratiometric fluorescence sensor for highly selective and sensitive detection of phycocyanin, Biosens. Bioelectron. 77 (2016) 624-630.

[29] N.W. Turner, E.V. Piletska, K. Karim, M. Whitcombe, M. Malecha, N. Magan, C. Baggiani, S.A. Piletsky, Effect of the solvent on recognition properties of molecularly imprinted polymer specific for ochratoxin A, Biosens. Bioelectron. 20 (2004) 1060-1067.

[30] Q. Yang, X. Wu, H. Peng, L. Fu, X. Song, J. Li, H. Xiong, L. Chen, Simultaneous phase-inversion and imprinting based sensor for highly sensitive and selective detection of bisphenol A, Talanta 176 (2018) 595-603.

Qian Yang received her BE degree of Food Science \& Engineering from Nanchang University, China, in 2016. And now she is studying for her master degree in School of Food Science \& Technology Nanchang University. Her current research interest is molecular imprinting fluorescence sensor for rapid detection of trace substance in food and environment.

Jinhua Li received her $\mathrm{PhD}$ in analytical chemistry from the Department of Chemistry of Hong Kong Baptist University, Hong Kong, in 2009. In the same year, she joined in Yantai Institute of Coastal Zone Research, Chinese Academy of Sciences, as an assistant professor. In 2015, she became an associate professor. Her current research interest focuses on preparation of novel molecular imprinting polymers and applications to sample pretreatment and chemo/biosensors.

Xiaoyan Wang received her PhD in School of Chemistry, Chemical Engineering and Materials Science, Shandong Normal University, Joint-Educated at Yantai Institute of Coastal Zone Research, Chinese Academy of Sciences, in 2017. Her current research interests focus on the preparation \& application of molecularly imprinted polymers in chromatographic separation and chemical sensors for analysis of typical pollutants.

Hailong Peng received her PhD in Food Science and Technology from the School of Food Science of Nanchang University, China, in 2014. In 2007, he joined in Department of Chemical Engineering, Nanchang University, as an assistant professor. In 2015, he became an associate professor. Her current research interest focuses on preparation of novel nanomaterials and its application in food and drug fields.

Hua Xiong received his B.Sc. from the Department of Biological and Agricultural Engineering, University of Arkansas, United States, in 1996. Now, he is a professor at Nanchang University and a permanent member of the State Key Lab of Food Science and Technology at Nanchang University. His current research interests are focused on food safety and food processing technology.

Lingxin Chen received his $\mathrm{PhD}$ degree in analytical chemistry from the Dalian Institute of Chemical Physics, Chinese Academy of Sciences, Dalian, in 2003. After 2 years of postdoctoral experience at the Department of Chemistry, Tsinghua University, Beijing, he joined first as a BK21 researcher and then as a research professor at the Department of Applied Chemistry, Hanyang University, Korea, in 2006. In 2009, as a professor, he joined the Yantai Institute of Coastal Zone Research, Chinese Academy of Sciences, Yantai. His research interests include the studies of novel properties of materials such as functionalized nanoparticles for developing nanoscale biochemical analysis methods and molecular imprinting-based sample pretreatment technology. 